\title{
EAl Endorsed Transactions

\section{A Pervasive Sensing Approach to Automatic Assessment of Trunk Coordination Using Mobile Devices}

\author{
Zilu Liang ${ }^{1,2, *}$, Yasuyuki Yoshida ${ }^{3}$, Nami Iino $^{3}$, Takuichi Nishimura ${ }^{3}$, Mario Alberto Chapa-Martell ${ }^{4}$, \\ and Satoshi Nishimura ${ }^{3}$
}

${ }^{1}$ Kyoto University of Advanced Science, Kyoto, Japan

${ }^{2}$ The University of Tokyo, Tokyo, Japan

${ }^{3}$ National Institute of Advanced Industrial Science and Technology, Tokyo, Japan

${ }^{4}$ CAC Corporation, Tokyo, Japan

\section{Abstract}

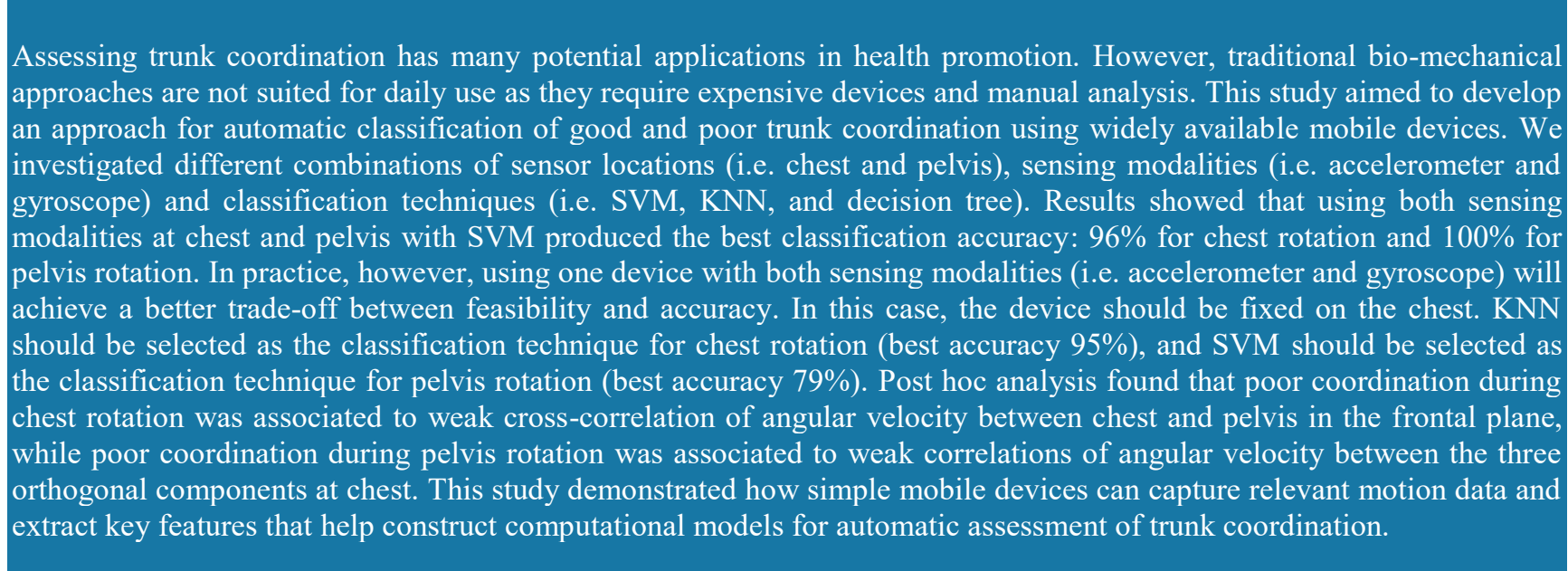

Keywords: trunk coordination; health promotion; pervasive computing; inertia measurement units (IMU); accelerometer; gyroscope; machine learning.

Received on 28 April 2018, accepted on 28 July 2018, published on 30 July 2018

Copyright (C) 2018 Zilu Liang et al., licensed to EAI. This is an open access article distributed under the terms of the Creative Commons Attribution licence (http://creativecommons.org/licenses/by/3.0/), which permits unlimited use, distribution and reproduction in any medium so long as the original work is properly cited.

doi: 10.4108/eai.13-7-2018.159604

"Corresponding author. Email:z.liang@cnl.t.u-tokyo.ac.jp

\section{Introduction}

Trunk strength is critical for the prevention of lower back pain and fall accident in elderly population $[1,2]$. Whereas people tend to equate trunk strength with the strength of trunk muscles, there is increasing evidence that the coordination of muscles, joints and ligaments is even more important in providing sufficient trunk strength and is yet not strongly associated to trunk muscle strength $[3,4]$.
Moreover, impaired coordination was found to be associated to many sickness including low back pain [5-7], stroke [8], and unilateral vestibular hypofunction [9]. To this end, trunk coordination ability can be used as an indicator of a person's physical health. Nevertheless, there is no tool supporting the assessment of dynamic coordination ability of trunk in daily life settings. Trunk coordination has mainly been studied in low back pain research [2, 5-7, 10-15]. Traditional approaches require the use of complicated and expensive motion analysis systems that can only be used in 
laboratories [5, 11]. It is not feasible to apply the same approaches to daily life settings.

This study aimed to develop an approach for automatic nominal assessment of trunk coordination ability using mobile devices. We sought for solutions at the intersection of pervasive sensing and machine learning, so that our methods could benefit anyone who has simple mobile devices such as smartphones, iPod and iPad. The accelerometers and gyroscopes embedded in these devices have enabled data-driven and ubiquitous approaches for motion tracking and analysis in free-living conditions $[9,16-$ 21]. These portable sensors allow for capturing motion data in all three planes of motion (i.e. sagittal plane, frontal plane, and transverse plane) and is thus suited for the analysis of trunk coordination [11]. We have previous experimented with other types of portable devices such as Nintendo Wii Board but failed to achieve high accuracy [22]. Our study differed from the previous studies in that we addressed the key issue of automating the assessment of coordination through machine learning based modelling. Machine learning has been widely used in health-related ubiquitous computing applications [21, 23-26]. The black-box approach enables us to model the complicated and high-dimensional relationships between features extracted from motion data and trunk coordination ability.

This study was conducted in three steps. We first defined two standard moves, i.e. chest rotation and pelvis rotation. These moves require smooth trunk-pelvis coordination and are easy to perform by the general population. Different from traditional biomechanical approaches that use static discrete tasks, we designed two continuous dynamic moves that provide spatial-temporal information to quantify trunk coordination.

Second, we conducted a data collection trial with a cohort of 21 participants. Two iPods with embedded accelerometers and gyroscopes were fixed on chest and on the back of pelvis to collect motion data. All moves were video recorded with the consent from participants. Since it is hard to obtain the ground truth of coordination ability, we chose to rely on the wisdom of experts. Three sports judges separately rated all the moves, and the majority of the three ratings for each move was used as the final label. The obtained datasets were split into training sets and testing sets for the machine learning process in the next step.

Third, we applied three machine learning techniques, i.e. support vector machine (SVM), k-nearest neighbour (KNN) and decision tree, to train the classification models. The performance of the classifiers was evaluated using four metrics: sensitivity, specificity, accuracy and balanced accuracy. We also investigated the effect of sensor location and sensing modality, and we identified the motion patterns indicating poor coordination through post hoc statistical analysis.

Our analysis revealed that using two devices (one fixed on the chest and the other fixed on the back of the pelvis) with SVM chosen as the machine learning technique produced the best classification accuracy on trunk coordination ability, i.e. $96 \%$ for chest rotation and $100 \%$ for pelvis rotation. In real situation, however, using one device with both sensing modalities (i.e. accelerometer and gyroscope) will achieve a better trade-off between feasibility and accuracy. In this case, the device should be fixed on the chest. KNN should be selected as the classification technique for chest rotation (best accuracy 95\%), and SVM should be selected as the classification technique for pelvis rotation (best accuracy 79\%) respectively. Post hoc analysis found statistically significant differences between good and poor coordination on the top five features. Poor coordination during chest rotation was associated to lower values of the maximal cross-correlation ( $\mathrm{y}$ component), the mean ( $\mathrm{x}$ component at pelvis), and the fifth fast Fourier transform (FFT) coefficient (y component at chest) of angular velocity, while poor coordination during pelvis rotation was associated to weaker correlations between the $x$ and the $y$ components, weaker correlations between the $x$ and the $z$ components, and weaker covariance between the $x$ and the $z$ component of the angular velocity of chest. We may infer that judges used different strategies in assessing coordination ability through the two standard moves. For chest rotation, attention was given to the movement of both chest and pelvis and their coordination. For pelvis rotation, attention was dominantly paid to the coordination of chest in three orthogonal directions.

This study demonstrated how simple mobile devices can capture relevant motion data and extract key features that help construct computational models for the automatic assessment of trunk coordination. The main contributions of this study are three-fold: (1) we developed a machine learning based pervasive sensing approach using widely available mobile devices; (2) we examined a number of combinations of sensor locations, sensing modalities and machine learning techniques and identified the best combinations; (3) we interpreted the machine learning models to understand the key features that characterise poor trunk coordination.

The rest of the paper is organized as follows. Section 2 provides a literature review on trunk coordination and mobile sensors for human movement analysis. Section 3 presents two standard moves, data collection protocol, and data analysis and modelling techniques. Performance evaluation of the classification models is presented in Section 4. We discuss the implication of the results within the landscape of previous studies in Section 5. The paper is concluded in Section 6.

\section{Related Work}

\subsection{Trunk Coordination}

Trunk coordination is an important aspect of trunk strength $[27,28]$ and has been intensively studied in low back pain research [2, 5-7, 10-15]. Different types of coordination have been investigated in previous studies, including headtrunk coordination [9], arm-trunk coordination [8], trunkpelvis coordination $[11,13,15]$, and trunk-lower limb coordination [29]. 
These studies focused on identifying kinematic differences in motions between individuals with pathological conditions and healthy individuals. Differences were found between normal group and low back pain group in average angle of flexion and average cycle velocity during dynamic trunk motion [12] and in the range of motion, velocity and acceleration during free dynamic oscillatory bending motion [30]. Coordination analysis demonstrates a reduction in relative motion between the pelvis and trunk in low-back-pain group at varied walking and running speed $[5,6,11]$. Moreover, trunk-pelvis was more in-phase for individuals with low back pain than those without low back pain [14].

Previous studies also illustrate the importance of evaluating all three planes of motion (i.e. sagittal plane, frontal plane and transverse plane as shown in Figure 1) simultaneously when studying trunk coordination $[11,31$ 33 ], as the spine is a complex structure exhibiting multiaxial motion during trunk rotational activities [34]. Indeed, the reduced ability to modulate coordination for pathological individuals was found both in frontal plane [11] and in transverse plane $[11,15]$.

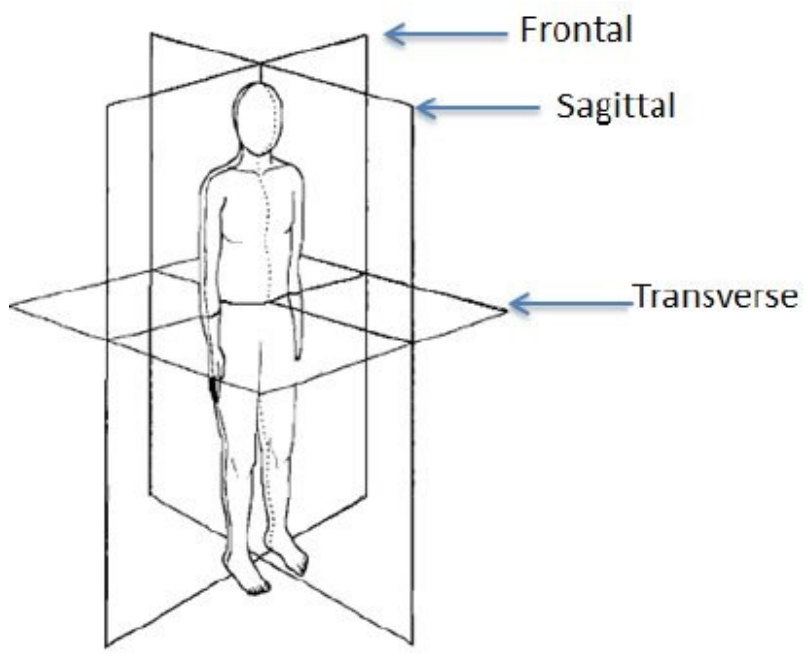

Figure 1. Cardinal planes of motion [35].

Quantitative assessment of trunk coordination has been an important topic in sport science and rehabilitation. While traditional biomechanical assessment of motion has typically used discrete measures such as peak excursions and forces, studies on coordination have mostly relied on continuous measures such as continuous relative phase (CRP) $[11,36-38]$. In addition, coordination variability defined as the difference between cycle standard deviation of CPR may be an effective indicator of motion quality and pathological conditions such as low back pain [7, 39, 40]. Previous studies have found decreased coordination variability in people with low back pain compared to healthy individuals [7].
Whereas the kinematic differences in trunk coordination between pathological and healthy individuals has been intensively studied, it remains unknown as to the differences between healthy individuals with good and poor coordination. The latter is an important topic to investigate because it produces insights into the prediction and the prevention of motor diseases such as low back pain and balance impairment. Hence, this study set out to fill in the knowledge gap by establishing a novel pervasive sensing approach to the automatic assessment of good and poor coordination among health people.

\subsection{Mobile Sensors in Human Movement Analysis}

Human motion analysis aims to quantitatively assess motor functions and motor abilities [41]. Traditional motion analysis relies on the use of optoelectronic systems (i.e. motion capture systems) that measure the kinematics and kinetics of body joints, which requires bulky and expensive equipment and can only be conducted in laboratory settings [42]. Technology advances in recent years has seen the rise of alternative measurement techniques using mobile sensors such as accelerometers and gyroscopes. These devices have multiple advantages, including low cost, light weight, and supporting pervasive measurement even in daily life settings [43].

A set of a mutually aligned three-orthogonal accelerometer and gyroscope is generally referred to as an inertial measurement unit (IMU). An IMU is widely available in modern portable devices such as smartphones and surfaces, making it easy to measure three-dimensional linear acceleration and angular velocity in daily life [16]. The accuracy and reliability of IMU has also been validated [44]. Many studies have used IMU for gait analysis [18, 45, 46]. Studies have examined people's the ability of maintaining upright balance during walking [47], step wise repeatability [48], gait resilience to external and internal perturbations [49], and the risks of fall [50]. Previous studies also identified various key metrics [51]. In the time domain, acceleration root mean square or standard deviation are the validated metrics for balance assessment [52]. In the frequency domain, the harmonic ratio (HR) is a valid metric for assessing the symmetry and rhythm of movements [53, 54], and the relative power of the first signal harmonic with respect to the total power of the signal is used to access the smoothness of gait [55].

Another important area using mobile sensors is the detection of daily activities [56-61] and sleep [23, 24, 62-65]. The main purposes include the detection of different types of activities in daily life, the modelling of daily activity patterns, the detection of sleep stages throughout a night, and the promotion of physical activities and sleep hygiene. This line of research has many applications to health promotion [20, 66, 67], rehabilitation [19, 68, 69] and sports training [70]. While it is relatively easy to distinguish among different postures such as sitting, standing, lying or motor activities [71, 72], quantifying the amount of 
activities is challenging. As a result, several populationspecific classification thresholds have been proposed and validated, including for toddlers [73], children [74, 75], youth [76], healthy adults [77, 78], and adults with neuromuscular control diseases [79-81].

This study presents a novel approach that nurtures the IMU embedded in widely available mobile devices to achieve the automatic assessment of trunk coordination ability in daily life settings. The proposed approach foresees a new application of these mobile sensors in the broad scope of health promotion. Our results produced rich implications for future research along the line of health-oriented pervasive sensing technologies.

\section{Materials and Methods}

The proposed approach provides a pervasive solution for anyone who wants to assess, track, and enhance their trunk coordination ability. The problem of assessing good and poor coordination was formulated into a classification problem, and thus standard machine learning techniques were applied. In what follows, we first present the two standard moves for assessing trunk coordination. We then describe the data collection protocol and data analysis and modelling techniques.

\subsection{Standard Moves}

Trunk coordination ability has been studied during walking, running, turning and bending [6, 37, 43, 46-48]. Whereas these moves may be effective medium to differentiate pathological population and healthy population in traditional biomechanical studies $[6,14,15]$, they may not serve the purpose of this study to distinguish the movement patterns of healthy people with good and poor coordination. The ideal standard moves should (1) require coordinated control of trunk muscle and joints, (2) involve rhythmic movements of trunk and lower extremities, (3) be easy and safe to perform by the general population.

After discussing with physical therapists and sports professionals, we selected the following two standard moves. The moves are illustrated in Figure 2. A demo video of the basic moves can be accessed at https://www.youtube.com/watch? $\mathrm{v}=7 \mathrm{cx} 4 \mathrm{KX} 3 \mathrm{JpMQ}$. It is worth noting that the tempo of pelvis rotation is quicker than that of chest rotation. Previous studies on trunk coordination during walking revealed distinct kinematic characteristics between slow and fast walk [6, 11, 33, 45, 46]. Therefore, the two standard moves allow for the assessment of trunk coordination under different rotation speed and thus will produce more insights.

- Standard Move 1: Chest Rotation. This move requires participants to repeatedly rotate their trunks from the left side to the right side to the end of the maximum angular displacement as possible. They were supposed to naturally shift their centre of gravity between legs while they rotate the trunk. As is shown in Figure 2 (a), chest rotation starts with feet apart at shoulder's width. The performer is required to rotate the upper body slowly while using hip joints as supporting points. The supporting foot should not be moved, and the heel of the other foot can be lifted. The small toe of the supporting foot should cling to the floor.

- Standard Move 2: Pelvis Rotation. This move requires participants to alternately lift one heel while naturally rotating the pelvis. As is shown in Figure 2 (b), pelvis rotation starts with feet together. The performer is required to switch the centre of gravity between two legs by alternating between bending and strengthening the knees, with the toes of the bending knee pointing the floor. The performer should keep the head stable and face forward.

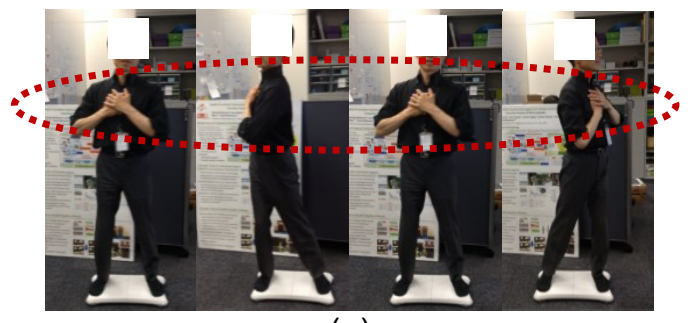

(a)

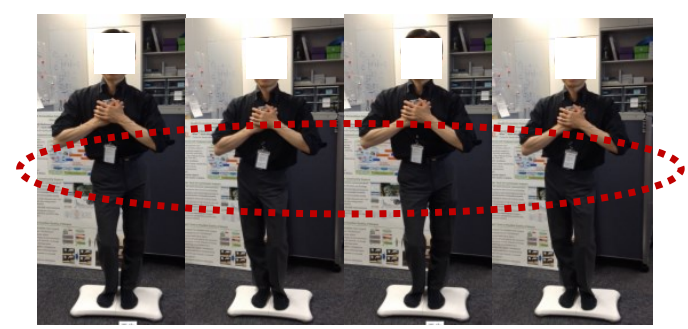

(b)

Figure 2. Standard moves: (a) chest rotation; (b) pelvis rotation.

\subsection{Data Collection Protocol}

\section{Participants}

We recruited 21 injury free adults (12 females and 9 males, year range: 24-65) for data collection. Participants were recruited from the National Institute of Advanced Industrial Science and Technology (AIST) and The University of Tokyo. No participant reported a major back or lower limb pathology, use of medication, or a history of neurologic disease that may influence neuromuscular control. This research was approved by the ethical committee of AIST. All participants signed informed consent before participating in the data collection trial.

\section{Devices and Sensing Modalities}

As depicted in Figure 3, two iPods were used to collect motion data concurrently. The devices were fixed in front of the chest and on the back of the pelvis to measure the 
movements of thorax and pelvis respectively. Previous studies have demonstrated the validity of these locations in studying motor control [10, 82-84].

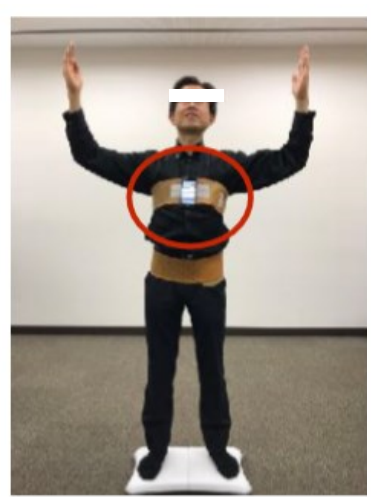

(a)

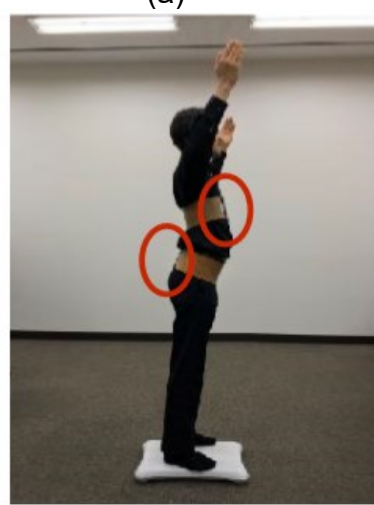

(c)

Figure 3. Sensor location in data collection trial: (a) front view; (b) back view; (c) side view. The two iPods were fixed on the chest and on the back of pelvis respectively using belts.

These two iPods were denoted as iPod ${ }^{\text {ch }}$ and iPodpel respectively. Each iPod has an embedded triaxial accelerometer and a triaxial gyroscope. These sensors enabled the iPods to measure acceleration and angular velocity in three orthogonal directions. The sampling frequencies of both sensing modalities were $50 \mathrm{~Hz}$, and the dynamic range of the accelerometer was $\pm 2.3 \mathrm{~g}$. The devices were rigidly fixed to the body segments using belts, with the sensors' reference system aligned with the anatomic axes of the segments where the sensors were attached. To make sure that data collected by the two iPods were aligned in time, we linked the two devices to a third iPod using a mobile application named Axis Visualizer that we developed in our previous study [85]. With the Axis Visualizer opened on all three iPods, we could start the measurement of iPod $^{\text {ch }}$ and iPodpel at exactly the same time by simply tapping the start button on the third iPod.

Measurement Protocol and Labelling
As is shown in Figure 3, the two iPods were fixed on the chest and on the back of the pelvis using belts. Participant was instructed to perform the standard moves by following the demo video (available at https://www.youtube.com/watch?v=7cx4KX3JpMQ). The data collection trial had three sessions. Each session consisted of performing chest rotation for 12 seconds and pelvis rotation for 12 seconds. Participants started with relaxing and adjusting the standing posture, followed by performing the three sessions with a 10 -second rest between sessions. All movements were video recorded.

After the trial, the videos were shown to three sports judges for rating. The rating was done independently, i.e. all judges were not aware of each other's assessment. The final rating was obtained using the following rule: a move was labelled as 1 only when at least two judges rate it as 1 (= poor coordination); or else the move was labelled as 0 (= good coordination).

\subsection{Data Analysis and Modelling Techniques}

Data from all the participants were used in the final analysis. Since the iPods collected raw data of acceleration and angular velocity in three planes of motion, the following 4 datasets were obtained for each standard move. In the following equations, $\boldsymbol{a}_{X}, \boldsymbol{a}_{Y}, \boldsymbol{a}_{Z}$ denote the $x, y$, and $z$ components of acceleration, and $\omega_{X}, \omega_{Y}, \omega_{Z}$ denote the $x, y$, and $z$ components of angular velocity.

- Dataset $\boldsymbol{A}^{\text {ch }}$ comprised the acceleration data in three orthogonal directions and the root sum of squares (RSS) collected by the accelerometer in iPod $^{\text {ch }}$.

$$
\begin{gathered}
\boldsymbol{A}^{c h}=\left\{\boldsymbol{a}_{X}^{c h}, \boldsymbol{a}_{Y}^{c h}, \boldsymbol{a}_{Z}^{c h}, \boldsymbol{a}_{R S S}^{c h}\right\} \\
\boldsymbol{a}_{R S S}^{c h}=\sqrt{\left(\boldsymbol{a}_{X}^{c h}\right)^{2}+\left(\boldsymbol{a}_{Y}^{c h}\right)^{2}+\left(\boldsymbol{a}_{Z}^{c h}\right)^{2}}
\end{gathered}
$$

- Dataset $A^{\text {pel }}$ comprised the acceleration data in three orthogonal directions and the RSS collected by the accelerometer in iPod ${ }^{\text {pel }}$.

$$
\begin{gathered}
\boldsymbol{A}^{p e l}=\left\{\boldsymbol{a}_{X}^{p e l}, \boldsymbol{a}_{Y}^{p e l}, \boldsymbol{a}_{Z}^{p e l}, \boldsymbol{a}_{R S S}^{p e l}\right\} \\
\boldsymbol{a}_{R S S}^{\text {pel }}=\sqrt{\left(\boldsymbol{a}_{X}^{\text {pel }}\right)^{2}+\left(\boldsymbol{a}_{Y}^{\text {pel }}\right)^{2}+\left(\boldsymbol{a}_{Z}^{\text {pel }}\right)^{2}}
\end{gathered}
$$

- Dataset $\Omega^{c h}$ comprised the angular velocity data in three orthogonal directions and the RSS collected by the gyroscope in iPod ${ }^{\text {ch }}$.

$$
\begin{gathered}
\boldsymbol{\Omega}^{c h}=\left\{\boldsymbol{\omega}_{X}^{c h}, \boldsymbol{\omega}_{Y}^{c h}, \boldsymbol{\omega}_{Z}^{c h}, \boldsymbol{\omega}_{R S S}^{c h}\right\} \\
\boldsymbol{\omega}_{R S S}^{c h}=\sqrt{\left(\boldsymbol{\omega}_{X}^{c h}\right)^{2}+\left(\boldsymbol{\omega}_{Y}^{c h}\right)^{2}+\left(\boldsymbol{\omega}_{Z}^{c h}\right)^{2}}
\end{gathered}
$$


- Dataset $\boldsymbol{\Omega}^{\text {pel }}$ comprised the angular velocity data in three orthogonal directions and the RSS collected by the gyroscope in iPod ${ }^{\text {ch }}$.

$$
\begin{gathered}
\boldsymbol{\Omega}^{p e l}=\left\{\boldsymbol{\omega}_{X}^{p e l}, \boldsymbol{\omega}_{Y}^{p e l}, \boldsymbol{\omega}_{Z}^{p e l}, \boldsymbol{\omega}_{R S S}^{p e l}\right\} \\
\boldsymbol{\omega}_{R S S}^{p e l}=\sqrt{\left(\omega_{X}^{p e l}\right)^{2}+\left(\omega_{Y}^{p e l}\right)^{2}+\left(\omega_{Z}^{p e l}\right)^{2}}
\end{gathered}
$$

\section{Data Pre-processing}

As was recommended in [86], the raw data of acceleration and angular velocity were smoothened using a median filter $(n=5)$. This process helped remove any abnormal noise spikes produced by the accelerometers and gyroscopes. The median-filtered acceleration data was then passed through a high-pass filter with cut-off frequency at $0.25 \mathrm{~Hz}$ to filter out the acceleration component due to gravity (GA) [87, 88]. The output of the high-pass filter was the pure body acceleration (BA) component.

\section{Feature Extraction and Selection}

Following standard machine learning process, we extracted time-domain and frequency-domain features from the preprocessed acceleration and angular velocity data. Since the standard moves involve repetitive motions, the slidingwindow approach widely used in activity recognition studies did not serve our purpose. Instead, the features were extracted from the whole time series data of a move.

Features in time domain were derived directly from the pre-processed raw data and were typically statistical measures. These features were found to be useful for activity recognition in previous studies [87, 89-92]. Features in frequency domain were derived from the fast Fourier transform (FFT) of the pre-processed raw data. We defined two feature sets. Feature Set I characterized the traits of data collected by each individual iPod and is summarized in Table 1. Feature Set II comprised features related to the cross-correlation between data collected by the two iPods to quantify the strength of coupling between the motion of the two body segments [93-97]. Feature Set II is summarized in Table 2.

One of the main themes of this paper was the analysis of different combinations of sensor location and sensing modality. We evaluated the performance of the nine combinations (thus denoted as nine methods) described in Table 3, where A and $\Omega$ represents the pre-processed acceleration and angular velocity datasets.

Feature selection was conducted to remove redundant features and to select the features with the strongest distinguishing power. Previous studies suggested that feature selection or dimension reduction was essential for improving the performance of machine learning techniques $[98,99]$. In this study, feature selection was performed according to the following process. First, the features with constant values in all samples were removed. Second, redundant features were removed through examining the pair-wise correlations of the features. If two features had a high correlation, we removed the one with larger mean absolute correlation as this feature had smaller variance. After that, we applied Random Forest Recursive Feature Elimination (RF-RFE) [100] to select the strongest features. The RF-RFE algorithm was a greedy optimization for identifying the subset of best features through multiple rounds of repetitions. In each repetition, the algorithm constructed a model using random forest algorithm, selected the features that produced the best accuracy set aside these features and then repeated the process with the rest of the features. This process stopped when all features in the datasets were exhausted. Thereafter the features were ranked according to when they were eliminated. Figure 4 presented an example of how the accuracy in repeated cross-validation changed as the number of features increased. The coordinates of the solid dot indicated the optimal number of features and the corresponding 10-fold cross-validation accuracy (repeated 3 times).

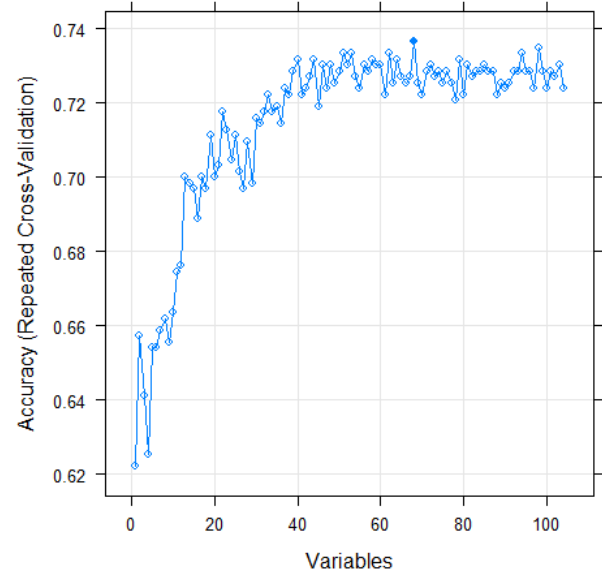

Figure 4. An example of random forest recursive feature elimination with automatic tuning of the number of features selected (10-fold cross-validation with 3 repetitions).

\section{Classification}

The problem of assessing trunk coordination ability was formulated into a classification problem. Three machine learning techniques were employed to achieve the goal: support vector machine (SVM), K-nearest neighbour (KNN), and decision tree. Following standard machine learning process, we split the collected dataset into training set and testing set. The training set comprised $70 \%$ of the samples in the original dataset, while the testing set comprised the rest $30 \%$. In total 27 classifiers ( 3 for each method) were trained using the corresponding training sets. 
Table 1. Features and denotations in Feature Set I.

\begin{tabular}{|c|c|c|c|}
\hline \multicolumn{2}{|c|}{ Time Domain } & \multicolumn{2}{|c|}{ Frequency Domain } \\
\hline Feature & Denotation & Feature & Denotation \\
\hline Maximum & $\begin{array}{l}\max X, \max Y, \max Z \\
\max R S S\end{array}$ & $\begin{array}{l}\text { First Five FFT } \\
\text { Coefficients }\end{array}$ & $\begin{array}{l}\mathrm{fftCoX} 1 \sim 5, \mathrm{fftCoY1} 5 \\
\mathrm{fftCoZ1} 5, \mathrm{fftCoRSS1} 5\end{array}$ \\
\hline Minimum & $\min X, \min Y, \min Z, \min R S S$ & Principle Frequency & $\begin{array}{l}\text { prinfreqX, prinfreq } Y \text {, prinfreqZ, } \\
\text { prinfreRSS }\end{array}$ \\
\hline Mean Value & $\begin{array}{l}\text { meanX, mean } Y, \min Z, \\
\text { meanRSS }\end{array}$ & Peak Frequency & $\begin{array}{l}\text { Peakfre } X X, \text { peakfreq } Y \\
\text { peakfreqZ, peakfreqRSS }\end{array}$ \\
\hline Standard Deviation & $s d X, s d Y, s d Z, s d R S S$ & Spectral Energy & seX, seY, seZ \\
\hline Kurtosis & kuX, kuY, kuZ, kuRSS & & \\
\hline Root Mean Square & $r m s X, r m s Y, r m s Z, r m s R S S$ & & \\
\hline Median Cross Zeros & $m c X, m c Y, m c Z$ & & \\
\hline Correlation X, Y, Z & $\operatorname{cor} X Y, \operatorname{cor} X Z, \operatorname{cor} Y Z$ & & \\
\hline
\end{tabular}

Table 2. Features and denotations in Feature Set II.

\begin{tabular}{ll}
\hline \multicolumn{2}{c}{ Time Domain } \\
\hline Feature & Denotation \\
Minimum value of cross-correlation between two iPods & $x$ CorMin, yCorMin, zCorMin, \\
Maximum value of cross-correlation between two iPods & $x$ CorMax, yCorMax, zCorMax \\
Lag of the minimum value of cross-correlation & $x$ lagMin, ylagMin, zlagMin, \\
Lag of the maximum value of cross-correlation & xlagMax, ylagMax, zlagMax \\
\hline
\end{tabular}

Table 3. Feature extraction for nine methods with varied sensor locations and sensing modalities.

\begin{tabular}{|c|c|c|c|c|}
\hline Methods & Sensor Location & Sensing Modality & Raw Data & Extracted Features \\
\hline \multicolumn{5}{|c|}{ Single device with single modality } \\
\hline Method-1 & Chest & Accelerometer & $A^{c h}$ & Feature Set I \\
\hline Method-2 & Chest & Gyroscope & $\boldsymbol{\Omega}^{c h}$ & Feature Set I \\
\hline Method-3 & Pelvis & Accelerometer & $A^{\text {pel }}$ & Feature Set I \\
\hline Method-4 & Pelvis & Gyroscope & $\Omega^{\text {pel }}$ & Feature Set I \\
\hline \multicolumn{5}{|c|}{ Single device with multiple modalities } \\
\hline Method-5 & Chest & $\begin{array}{l}\text { Accelerometer \& } \\
\text { gyroscope }\end{array}$ & $A^{c h}, \Omega^{c h}$ & Feature Set I \\
\hline Method-6 & Pelvis & $\begin{array}{l}\text { Accelerometer \& } \\
\text { gyroscope }\end{array}$ & $A^{p e l}, \Omega^{p e l}$ & Feature Set I \\
\hline \multicolumn{5}{|c|}{ Multiple devices with single modality } \\
\hline Method-7 & Chest, pelvis & Accelerometer & $A^{c h}, A^{p e l}$ & Feature Set I \& II \\
\hline Method-8 & Chest, pelvis & Gyroscope & $\boldsymbol{\Omega}^{c h}, \boldsymbol{\Omega}^{p e l}$ & Feature Set I \& II \\
\hline \multicolumn{5}{|c|}{ Multiple devices with multiple modalities } \\
\hline Method-9 & Chest, pelvis & $\begin{array}{l}\text { Accelerometer \& } \\
\text { gyroscope }\end{array}$ & $A^{c h}, A^{p e l}, \Omega^{c h}, \Omega^{p e l}$ & Feature Set I \& II \\
\hline
\end{tabular}




$$
\begin{gathered}
\text { Sensitivity }=\frac{T P}{T P+F N^{\prime}} \\
\text { Specificity }=\frac{T N}{T N+F P^{\prime}} \\
\text { Accuracy }=\frac{T P+T N}{T P+T N+F P+F N^{\prime}} \\
\text { Balanced Accuracy }=\frac{\text { Sensitivity }+ \text { Specificity }}{2},
\end{gathered}
$$

The parameter tuning for each classification technique was conducted in the following way. For SVM, the best value of parameters $\mathrm{C}$ and $\gamma$ were decided using grid search with radial basis function (RBF) kernel. For $\mathrm{KNN}$ and decision tree, the best value of parameter $\mathrm{k}$ and $\mathrm{cp}$ were decided through attempting different values. In addition, the performance of two split methods, information gain and Gini impurity were also investigated for decision tree [101]. The value corresponding to the highest accuracy (10-fold cross validation with 3 times repetition) was selected as the optimal values for the parameters.

The performance of the classifiers was then tested on the corresponding testing sets and evaluated using the following metrics defined in Equation (1)-(4). The TP, TN, FP, and FN represent true positive, true negative, false positive and false negative respectively. The sensitivity and specificity are the ability of a classifier to correctly detect good and poor coordination respectively [102]. Accuracy is the ability of a classifier to differentiate good and poor coordination [103], while balanced accuracy also counts in the imbalance of good and poor samples in the testing sets (where there were more poor samples) [104]. Balanced accuracy was chosen over F-score because there were more positive samples than negative ones.

\section{Post-hoc Analysis}

The output of a machine learning process was a trained classifier that performs nominal assessment of trunk coordination ability. However, the relationships between the key features and the corresponding classification result remained unclear, which hinders our understanding on the feature-wise difference between good and poor coordination. To address this problem, we conducted post-hoc analysis using Wilcoxon Signed Rank test [105] to examine if there was statistically significant difference on the mean value of a feature between good and poor coordination. Box-andWhisker [106] plots were also used to provide an intuitive display of the distribution of the feature values based on minimum, first quartile, median, third quartile, and maximum.

\section{Performance Evaluation}

This section presents the computation results and the performance evaluation of the nine methods described in
Table 3. The final datasets comprised motion data from 21 participants. Two datasets were collected in the trial, i.e. dataset-CR (chest rotation) and dataset-PR (pelvis rotation), with 63 samples in each dataset. The assessment by three judges yielded 32 (out of 63) samples of poor coordination during chest rotation, and 45 (out of 63) samples of poor coordination during pelvis rotation.

The $\mathrm{R}$ programming environment [107] was used for feature selection and classification. Features were extracted for each method. Depending on the number of devices and sensing modalities used in the method, the number of features varied. We investigated the effect of the following factors on classification accuracy: sensor location (chest versus pelvis), sensor modality (accelerometer versus gyroscope) and classification technique (SVM, KNN, and decision tree).

\subsection{Feature Selection}

Since the feature set for each method in Table 3 varied with respect to the number of devices and sensing modalities, we conducted feature selection for each method separately following the process described in Section 2.3.

Firstly, we removed the features that had the constant values in all samples in the datasets. These features include the minimum of $\boldsymbol{a}_{R S S}^{c h}, \boldsymbol{a}_{R S S}^{\text {pel }}, \boldsymbol{\omega}_{R S S}^{c h}$, and $\boldsymbol{\omega}_{R S S}^{\text {pel }}$, which were constantly zero. Secondly, we removed the redundant features based on pair-wise correlations. The number of redundant features varied in each method. Third, the Random Forest Recursive Feature Elimination (RF-RFE) [100] was applied to select the strongest features. The optimal number of features was selected via 10 -fold cross validation with 3 times of repetition. The number of features that remained after recursive feature elimination and the top five features were summarized in Table 4 (chest rotation) and Table 5 (pelvis rotation). The top five features were listed in descending order in terms of importance. We confirmed that using the remaining features produced better results than using the original feature set.

The results showed that important features were selected from both the time domain and the frequency domain. The number of selected features generally increased as the number of devices and sensing modalities increased. All iPods (i.e. chest and pelvis) and sensing modalities (i.e. accelerometer and gyroscope) contributed strong features to the classification of good and poor coordination. Most of the top five features were time-domain features, especially for pelvis rotation. Under the category of single device with multiple modalities for chest rotation in Table 4, we found that the iPod on the chest relied more on the sensing modality of gyroscope because all the top five features were extracted from the angular velocity data. In contrast, the iPod on the back of the pelvis relied more on the sensing modality of accelerometer. With respect to the feature selection for pelvis rotation, we found that the top five features selected in Method-8 and Method-9 were dominantly extracted from the accelerometer and gyroscope on the chest, indicating that the judges may have paid more 
attention to the movement of chest in assessing coordination during pelvis rotation.

\subsection{Parameter Tuning and Training}

The original datasets were split into training sets (42 samples) and testing sets (21 samples). Three classification techniques (i.e. SVM, KNN, and decision tree) were applied to train classifiers for automatic classification on good and poor coordination. We obtained the optimal values of the parameters for each classification technique following the process described in 2.3.4, with 3 repetitions of 10 -fold cross validation using the training sets. At each of the folds, the training set was partitioned by randomly selecting approximately $70 \%$ sample for training and $30 \%$ samples for testing. The optimal values of the parameters in the trained classifiers were selected based on the average classification accuracy over 3 repetitions, which were summarized in Table 6 (chest rotation) and Table 7 (pelvis rotation).

Table 4. Features selection in different methods for chest rotation.

\begin{tabular}{|c|c|c|}
\hline Methods & $\begin{array}{c}\text { Number of Selected } \\
\text { Features }\end{array}$ & Top 5 Features \\
\hline \multicolumn{3}{|c|}{ Single device with single modality (originally 65 features) } \\
\hline Method-1 & 29 & $\operatorname{corY} Z_{a}^{c h}, f f t C o Y 4_{a}^{c h}$, peakr $Y_{a}^{c h}$, prinfreq ${ }_{a}^{c h}$, peakrRSS $S_{a}^{c h}$ \\
\hline Method-2 & 15 & $\operatorname{seX}_{g}^{c h}, s d X_{g}^{c h}, s d X_{g}^{c h}, r m s X_{g}^{c h}, s d Z_{g}^{c h}$, meanZ $_{q}^{c h}$ \\
\hline Method-3 & 12 & $r m s Z_{a}^{\text {pel }}$, mean $Z_{a}^{\text {pel }}$, meanRSS $S_{a}^{\text {pel }}, k u R S S_{a}^{\text {pel }}, s d Z_{a}^{\text {pel }}$ \\
\hline Method-4 & 19 & $m c X_{g}^{p e l}, m c Z_{g}^{p e l}, \operatorname{covX} Z_{g}^{p e l}, s d Z_{g}^{\text {pel }}, r m s Z_{g}^{\text {pel }}$ \\
\hline \multicolumn{3}{|c|}{ Single device with multiple modalities (originally 130 features) } \\
\hline Method-5 & 78 & fftCoZ $4_{g}^{c h}, \min Z_{g}^{c h}, \operatorname{corY} Z_{a}^{c h}, \operatorname{covY} Z_{g}^{c h}, f f t C o Y 5_{g}^{c h}$ \\
\hline Method-6 & 31 & meanRSS $S_{a}^{p e l}, s d Y_{a}^{p e l}, k u R S S_{a}^{p e l}, r m s X_{a}^{p e l}, r m s Y_{a}^{p e l}$ \\
\hline \multicolumn{3}{|c|}{ Multiple devices with single modality (originally 142 features) } \\
\hline Method-7 & 111 & $\operatorname{corY} Z_{a}^{c h}$, peakrX $X_{a}^{\text {pel }}$, fftCoY $4_{a}^{c h}$, prinfreq $q_{a}^{c h}$, peakr $r_{a}^{c h}$ \\
\hline Method-8 & 101 & $\operatorname{yCorMax}_{g}, \mathrm{fftCoZ}_{g}^{c h}$, meanX $_{g}^{\text {pel }}$, zCorMax $_{g}, \mathrm{fftCoY}_{g}^{\mathrm{ch}}$ \\
\hline \multicolumn{3}{|c|}{ Multiple devices with multiple modalities (originally 284 features) } \\
\hline Method-9 & 183 & $y \operatorname{CorMax}_{g}, \mathrm{fftCoZ}_{g}^{\text {ch }}$, meanX $_{g}^{\text {pel }}, \mathrm{zCorMax}_{g}, \mathrm{fftCoY}_{g}^{\text {ch }}$ \\
\hline
\end{tabular}

Table 5. Features selection in different methods for pelvis rotation.

\begin{tabular}{|c|c|c|}
\hline Methods & $\begin{array}{c}\text { Number of Selected } \\
\text { Features }\end{array}$ & Top 5 Features \\
\hline \multicolumn{3}{|c|}{ Single device with single modality (originally 65 features) } \\
\hline Method-1 & 20 & 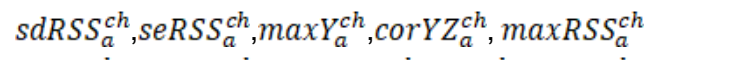 \\
\hline Method-2 & 17 & $r m s Z_{g}^{c h}$, meanZ $_{g}^{c h}, f f t C o Z 5_{g}^{c h}, s d Z_{g}^{c h}, r m s X_{g}^{c h}$ \\
\hline Method-3 & 11 & $m s Z_{a}^{p e l}, f f t C o Z 5_{a}^{p e l}, \max Z_{a}^{p e l}, s d Z_{a}^{p e l}, r m s X_{a}^{p e l}$ \\
\hline Method-4 & 14 & $\operatorname{meanZ_{g}^{\text {pel}}}, f f t C o Z 5_{g}^{\text {pel }}, r m s Z_{g}^{\text {pel }}, s d Z_{g}^{\text {pel }}, s d X_{g}^{\text {pel }}$ \\
\hline \multicolumn{3}{|c|}{ Single device with multiple modalities (originally 130 features) } \\
\hline Method-5 & 41 & $\operatorname{sdRSS} S_{a}^{c h}, \operatorname{corX} Y_{g}^{c h}, \operatorname{corX} Z_{g}^{c h}, \operatorname{seRSS} S_{a}^{c h}, f f t C o R S S 3_{g}^{c h}$ \\
\hline Method-6 & 68 & $f f t C o X 3_{g}^{p e l}, \operatorname{mcX}_{a}^{p e l}, \operatorname{maxRSS_{g}^{pel}}, \operatorname{minY}_{g}^{\text {pel }}, f f t C o Z 4_{a}^{p e l}$ \\
\hline \multicolumn{3}{|c|}{ Multiple devices with single modality (originally 142 features) } \\
\hline Method-7 & 78 & $\operatorname{sdRSS} S_{a}^{c h}, \operatorname{seRSS}_{a}^{c h}, z \operatorname{CorMax}{ }_{a}, \operatorname{mcX}_{a}^{p e l}, \operatorname{corY} Z_{a}^{c h}$ \\
\hline Method-8 & 45 & $\operatorname{corX} Y_{g}^{c h}, \operatorname{covY} Z_{g}^{c h}, \operatorname{cor} X Z_{g}^{c h}, \operatorname{covX} Z_{g}^{c h}, \operatorname{cor} Y Z_{g}^{c h}$ \\
\hline \multicolumn{3}{|c|}{ Multiple devices with multiple modalities (originally 284 features) } \\
\hline Method-9 & 111 & $\operatorname{corXY}_{g}^{c h}, z \operatorname{CorMax}{ }_{a}, \operatorname{covX} Z_{g}^{c h}, \operatorname{sdRSS} S_{a}^{c h}, \operatorname{corX} Z_{g}^{c h}$ \\
\hline
\end{tabular}

SVM achieved the best cross-validation accuracy for both moves. For chest rotation, the training accuracy of SVM was generally the best among the three techniques, while decision tree was the weakest technique among the three. For pelvis rotation, SVM and KNN produced similar training performance and were both better than decision tree. 


\subsection{Performance of Classification Models}

In total 9 classification models were obtained for chest rotation. The performance of the models was examined using the corresponding test datasets. The sensitivity, specificity, accuracy and balanced accuracy are summarized in Table 8. As expected, Method-9 (accelerometers and gyroscopes on chest and pelvis) with SVM achieved the best classification accuracy among all methods with a balanced accuracy of 0.96 . Despite using only one device, Method-2 (gyroscope on chest) with SVM and Method-5 (accelerometer and gyroscope on chest) with KNN achieved equivalently good classification accuracy as method Method-9. Regardless of the classification technique applied, sensors on the chest produced stronger features than those on the pelvis, and gyroscopes were more effective than accelerometer in classifying coordination. Using feature sets from different data sources, the best accuracy of SVM and KNN were similar and were approximately $95 \%$, whereas the best accuracy of decision tree was no more than $73 \%$. The trained decision tree that achieved the best classification accuracy on chest rotation is shown in Figure 5(a).

Nine classifiers were obtained for pelvis rotation in a similar manner. The performance of the classifiers for each method was examined on the corresponding test datasets. The sensitivity, specificity, accuracy and balanced accuracy are summarized in Table 9. Since the dataset of pelvis rotation was imbalanced with more positive observations, the disparity between accuracy and balanced accuracy was obvious especially when decision tree technique was applied. Regardless of using two iPods and two sensing modalities, Method-9 did not produce the best performance with any classification technique. In comparison, Method-8 (gyroscope on both chest and pelvis) with SVM achieved the best performance (100\% balanced accuracy), followed by Method-3 (accelerometer on pelvis) with KNN achieving a balanced accuracy of $72 \%$. SVM and KNN produced similar classification accuracy for all other methods, whereas the performance of decision tree was no better than random guess in most cases when counting in the imbalance of positive and negative samples. Decision tree only achieved slightly better performance with Method-2 (gyroscope on chest) and the trained decision tree is shown in Figure 5(b).

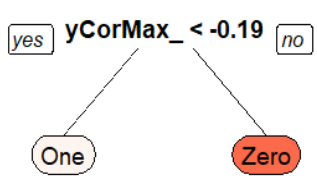

(a)

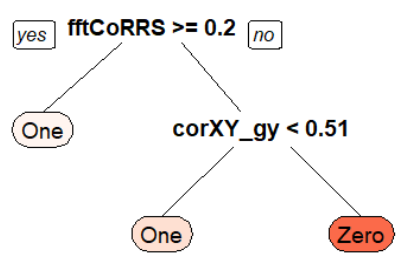

(b)
Figure 5. The trained decision trees that achieved the best performance on classifying good and poor coordination during (a) chest rotation and (b) pelvis rotation.

\subsection{Post-hoc Analysis}

To understand the relationships between the features and classification results, we conducted post hoc analysis using Wilcoxon Signed Rank (WSR) test [105]. WSR was selected in place of parametric statistical hypothesis tests such as ANOVA test due to the non-normality of the features indicated by the Shapiro-Wilk test [108]. Box-andWhisker plots were also used to compare the distribution of the feature values between good and poor coordination. The post-hoc analysis was conducted on the top five features of the best classification methods, as they were the most important and interesting features.

Table 6. Tuned parameters and best cross-validation accuracy of classification technique for chest rotation.

\begin{tabular}{|c|c|c|c|c|c|c|c|c|c|}
\hline & \multicolumn{4}{|c|}{ SVM } & \multicolumn{2}{|c|}{ KNN } & \multicolumn{3}{|c|}{ Decision Tree } \\
\hline & $\mathrm{C}$ & $\gamma$ & Num of SV & Best Acc & $\mathrm{k}$ & Best Acc & $\begin{array}{l}\text { Complexity } \\
\text { Parameter }\end{array}$ & $\begin{array}{c}\text { Split } \\
\text { Method }\end{array}$ & $\begin{array}{l}\text { Best } \\
\text { Acc }\end{array}$ \\
\hline Method-1 & 8 & 0.001 & 35 & 0.72 & 7 & 0.69 & 0.44 & Gini & 0.58 \\
\hline Method-2 & 256 & 0.001 & 20 & 0.91 & 5 & 0.74 & 0.28 & Gini & 0.61 \\
\hline Method-3 & 64 & 0.001 & 28 & 0.76 & 5 & 0.63 & 0.15 & Gini & 0.54 \\
\hline Method-4 & 1 & 0.01 & 40 & 0.72 & 5 & 0.62 & 0.04 & Gini & 0.57 \\
\hline Method-5 & 2 & 0.01 & 40 & 0.67 & 5 & 0.74 & 0.55 & Gini & 0.66 \\
\hline Method-6 & 4 & 0.001 & 41 & 0.70 & 5 & 0.60 & 0.15 & Gini & 0.51 \\
\hline Method-7 & 8 & 0.001 & 38 & 0.74 & 9 & 0.61 & 0.42 & Gini & 0.50 \\
\hline Method-8 & 1 & 0.01 & 42 & 0.76 & 5 & 0.68 & 0.21 & Gini & 0.57 \\
\hline Method-9 & 5 & 0.001 & 40 & 0.79 & 5 & 0.70 & 0.51 & Gini & 0.59 \\
\hline
\end{tabular}


Table 7. Tuned parameters and best cross-validation accuracy of classification technique for pelvis rotation.

\begin{tabular}{|c|c|c|c|c|c|c|c|c|c|}
\hline & \multicolumn{4}{|c|}{ SVM } & \multicolumn{2}{|c|}{ KNN } & \multicolumn{3}{|c|}{ Decision Tree } \\
\hline & C & $\gamma$ & $\begin{array}{c}\text { Num of } \\
\text { SV }\end{array}$ & $\begin{array}{l}\text { Best } \\
\text { Acc }\end{array}$ & $\mathrm{k}$ & $\begin{array}{l}\text { Best } \\
\text { Acc }\end{array}$ & $\begin{array}{l}\text { Complexity } \\
\text { Parameter }\end{array}$ & $\begin{array}{c}\text { Split } \\
\text { Method }\end{array}$ & $\begin{array}{l}\text { Best } \\
\text { Acc }\end{array}$ \\
\hline Method-1 & 0.001 & 0.000001 & 20 & 0.76 & 23 & 0.76 & 0.2 & Gini & 0.50 \\
\hline Method-2 & 5 & 1 & 39 & 0.71 & 7 & 0.80 & 0.27 & Information & 0.61 \\
\hline Method-3 & 64 & 0.001 & 22 & 0.81 & 5 & 0.82 & 0.25 & Gini & 0.50 \\
\hline Method-4 & 100 & 0.0001 & 30 & 0.74 & 15 & 0.72 & 0.42 & Gini & 0.50 \\
\hline Method-5 & 4 & 0.01 & 24 & 0.93 & 5 & 0.83 & 0.44 & Gini & 0.50 \\
\hline Method-6 & 256 & 0.0001 & 23 & 0.84 & 5 & 0.83 & 0.25 & Gini & 0.50 \\
\hline Method-7 & 2 & 0.01 & 39 & 0.74 & 5 & 0.75 & 0.31 & Gini & 0.50 \\
\hline Method-8 & 256 & 0.001 & 20 & 0.91 & 13 & 0.76 & 0.30 & Gini & 0.50 \\
\hline Method-9 & 2 & 0.01 & 40 & 0.81 & 5 & 0.73 & 0.42 & Gini & 0.50 \\
\hline
\end{tabular}

Table 8. Performance of classifiers for chest rotation.

\begin{tabular}{|c|c|c|c|c|c|c|c|c|c|c|c|c|}
\hline & \multicolumn{4}{|c|}{ SVM } & \multicolumn{4}{|c|}{$\mathrm{KNN}$} & \multicolumn{4}{|c|}{ Decision Tree } \\
\hline & Sen & Spe & Acc & Bal Acc & Sen & Spe & Acc & Bal Acc & Sen & Spe & Acc & Bal Acc \\
\hline Method-1 & 0.43 & 1.00 & 0.62 & 0.71 & 0.36 & 1.00 & 0.57 & 0.68 & 0.43 & 0.86 & 0.57 & 0.64 \\
\hline Method-2 & 0.93 & 1.00 & 0.95 & 0.96 & 0.57 & 1.00 & 0.71 & 0.79 & 0.43 & 0.71 & 0.52 & 0.57 \\
\hline Method-3 & 0.58 & 0.67 & 0.62 & 0.63 & 0.83 & 0.56 & 0.71 & 0.69 & 0.33 & 0.78 & 0.52 & 0.56 \\
\hline Method-4 & 0.90 & 0.50 & 0.70 & 0.70 & 0.90 & 0.50 & 0.70 & 0.70 & 0.50 & 0.30 & 0.40 & 0.40 \\
\hline Method-5 & 1.00 & 0.80 & 0.90 & 0.90 & 1.00 & 0.90 & 0.95 & 0.95 & 0.45 & 0.40 & 0.43 & 0.43 \\
\hline Method-6 & 0.58 & 0.75 & 0.65 & 0.67 & 0.83 & 0.75 & 0.80 & 0.79 & 0.42 & 0.63 & 0.50 & 0.52 \\
\hline Method-7 & 0.46 & 0.63 & 0.52 & 0.54 & 0.61 & 0.75 & 0.67 & 0.68 & 0.31 & 0.50 & 0.38 & 0.40 \\
\hline Method-8 & 0.89 & 0.64 & 0.75 & 0.76 & 0.44 & 0.91 & 0.70 & 0.68 & 0.56 & 0.91 & 0.75 & 0.73 \\
\hline Method-9 & 0.92 & 1.00 & 0.95 & 0.96 & 0.62 & 0.71 & 0.65 & 0.66 & 0.77 & 0.28 & 0.60 & 0.53 \\
\hline
\end{tabular}

Table 9. Performance of classifiers for pelvis rotation.

\begin{tabular}{lrrrrrrrrrrrrr}
\hline & \multicolumn{4}{c}{ SVM } & \multicolumn{4}{c}{ KNN } & \multicolumn{4}{c}{ Decision Tree } \\
\cline { 2 - 13 } & Sen & Spe & Acc & Bal Acc & Sen & Spe & Acc & Bal Acc & Sen & Spe & Acc & Bal Acc \\
\hline Method-1 & 1.00 & 0.00 & 0.62 & 0.50 & 1.00 & 0.00 & 0.62 & 0.50 & 1.00 & 0.00 & 0.62 & 0.50 \\
Method-2 & 0.93 & 0.29 & 0.71 & 0.61 & 0.93 & 0.29 & 0.71 & 0.61 & 0.93 & 0.29 & 0.71 & 0.61 \\
Method-3 & 0.80 & 0.33 & 0.67 & 0.57 & 0.93 & 0.50 & 0.81 & 0.72 & 1.00 & 0.00 & 0.71 & 0.50 \\
Method-4 & 0.93 & 0.17 & 0.71 & 0.55 & 0.87 & 0.17 & 0.67 & 0.52 & 1.00 & 0.00 & 0.71 & 0.50 \\
Method-5 & 0.92 & 0.67 & 0.81 & 0.79 & 1.00 & 0.22 & 0.67 & 0.61 & 1.00 & 0.00 & 0.57 & 0.50 \\
Method-6 & 1.00 & 0.20 & 0.62 & 0.60 & 1.00 & 0.10 & 0.57 & 0.55 & 1.00 & 0.00 & 0.52 & 0.50 \\
Method-7 & 1.00 & 0.60 & 0.90 & 0.80 & 1.00 & 0.40 & 0.856 & 0.70 & 1.00 & 0.00 & 0.76 & 0.50 \\
Method-8 & 1.00 & 1.00 & 1.00 & 1.00 & 1.00 & 0.14 & 0.70 & 0.57 & 1.00 & 0.00 & 0.65 & 0.50 \\
Method-9 & 1.00 & 0.40 & 0.85 & 0.70 & 1.00 & 0.20 & 0.80 & 0.60 & 1.00 & 0.00 & 0.75 & 0.50 \\
\hline
\end{tabular}

For chest rotation, all the features of interest were extracted from angular velocity of chest and pelvis. The WSR test showed that there were significant differences between good and poor coordination on the mean values of the following features: the maximal cross-correlation of the $y$ component between chest and pelvis $(p<0.001)$, the 
fourth FFT coefficient of the $z$ component of chest movement $(p=0.01)$, the mean value of the $x$ component of pelvis movement $(p=0.003)$, the maximal cross-correlation of the $z$ component between chest and pelvis $(p=0.003)$, and the fifth FFT coefficient of the $y$ component of chest movement $(p=0.002)$.

For pelvis rotation, all features of interest were extracted from angular velocity data measured on the chest. Significant differences between good and poor coordination were found on the mean values of the correlations between the $x$ and $y$ components $(p=0.002)$, between the $x$ and $z$ components $(p=0.02)$, and between the $y$ and $z$ components $(p=0.01)$. There were also differences on the covariance between the $y$ and $z$ components $(p=0.03)$, and between the $x$ and $z$ components $(p=0.04)$.

As is shown in Figure 6 (chest rotation) and Figure 7 (pelvis rotation), the Box-and-Whisker plots provide intuitive visual display on the distribution of the feature values between good and poor coordination. The thick lines, bot edges and whiskers represent the median, the 25-75\% quartile range and the overall range respectively. Compared to participants with good coordination, the movements of participants with poor coordination during chest ration were characterized by lower values of the maximal crosscorrelation in the $y$ component of the angular velocity between chest and pelvis in Figure 6(a), lower values of the mean of the $x$ component of the angular velocity of pelvis in Figure 6(c), and lower values of the fifth FFT coefficient of the $y$ component of the angular velocity of chest in Figure 6(e). Although there were significant differences between good and poor coordination on the mean values of the other two features, the Box-and-Whisker plots demonstrated large overlap of the $25-75 \%$ quartile range as is shown in Figure 6(b) and Figure 6(d). Therefore, these two features were not used to interpret the classification results. As for pelvis rotation, poor coordination was associated to weaker correlation between the $x$ and the $y$ components of the angular velocity of chest in Figure 7(a), weaker correlation between the $x$ and the $z$ components of the angular velocity of chest in Figure 7(c), and weaker covariance between the $x$ and $z$ components of the angular velocity of chest in Figure 7(d). For the same reason of overlapping in distribution, the other two features were not used to interpret the classification results.

\subsection{Participants Feedback}

At the end of the data collection trial, the participants were asked to provide feedback on the two standard moves and the demo video. All participants acknowledged that the devices were light to wear and the demo video was very easy to follow. They acknowledged that chest rotation was easy to perform because it resembled many movements in daily life. However, five participants found pelvis rotation awkward to perform, which echoed the fact that there were more positive samples in the datasets. As for the reasons, some participants mentioned that "it's not a common movement in daily life", "you have to rotate pelvis and tap the toe at the same time" and "it looks like walking, but you are not walking forward". These participants encountered difficulties in coordinating trunk and pelvis when performing pelvis rotation. Four participants pointed out that the side view of pelvis rotation in the demo video was very helpful for them to understand the correct form of this move. Given the challenge of performing pelvis rotation for some participants, it would be interesting to investigate the possibility of using pelvis rotation as an exercise for enhancing trunk-pelvis coordination in the context of community-based health promotion.

\section{Discussion}

We have analysed the performance of the proposed approach for automatic assessment of trunk coordination during two standard moves. The results showed that the best classification accuracy was achieved for the two standard moves when multiple devices were used with SVM chosen as the machine learning technique. The best methods achieved a Balanced Accuracy of 96\% (Method-9) and $100 \%$ (Method-8) respectively for chest rotation and pelvis rotation, with satisfying performance on correctly detecting positive samples (Sensitivity) and negative samples (Specificity) as well. Feature selection indicated that the cross-correlations between the movement of chest and that of pelvis were important features in assessing trunk coordination. For chest rotation, comparable performance was achieved by using only one device on the chest, either with one sensing modality of gyroscope (Method-2) or with both gyroscope and accelerometer (Method-5). These methods may be more feasible and appealing in real situations as they only rely on one device. As for sensor location, the results revealed that the assessment model based on the motion data of chest achieved better accuracy than those based on the motion data of pelvis.

It is also reasonable to assume that both sensing modalities are available in real situation as most mobile devices have embedded gyroscope as well as accelerometer. Therefore, using two sensing modalities on chest (Method5 ) is the most suitable method that balances the trade-off between feasibility and accuracy. In the meanwhile, KNN and SVM should be selected as the classification technique for chest rotation and pelvis rotation respectively so that a Balanced Accuracy of $95 \%$ and $79 \%$ could be achieved. In the meantime, the top five features selected in the best methods were all extracted from angular velocity data, we may infer that gyroscope may be more effective than accelerometer in capturing useful information for assessing trunk coordination. This result is in line with the practice in movement science where angular oscillations have been routinely used to study body movements [36].

Comparing the three machine learning techniques, SVM and $\mathrm{KNN}$ produced comparable performance, whereas decision tree was weaker than the other two. However, interpreting the classification rules of the trained decision tree was straightforward. As is shown in Figure 5, the classification of good and poor coordination relied only on 
one feature, i.e. the maximal cross-correlation of the $y$ component of the angular velocity between the chest and the pelvis. In other words, a move will be considered as an indicator of poor coordination if the previous-mentioned maximal cross-correlation is negative. In comparison, identifying and interpreting the relationships between the input features and the final classification outcomes for SVM and $\mathrm{KNN}$ required more complicated post-hoc analysis.

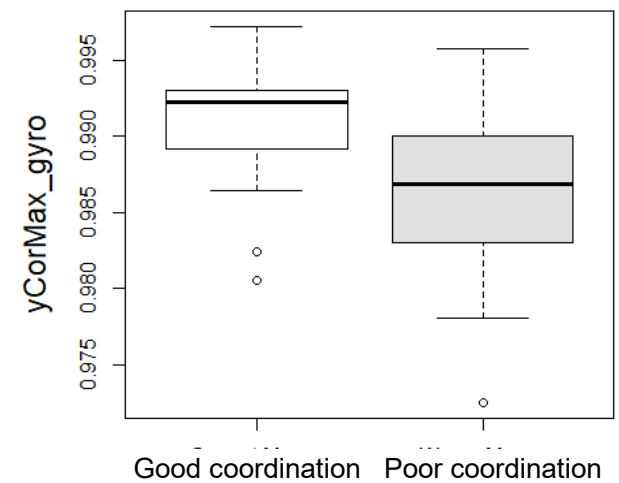

(a)

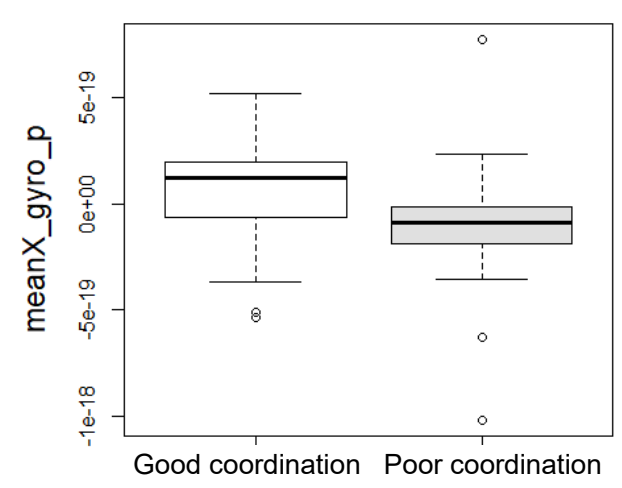

(c)

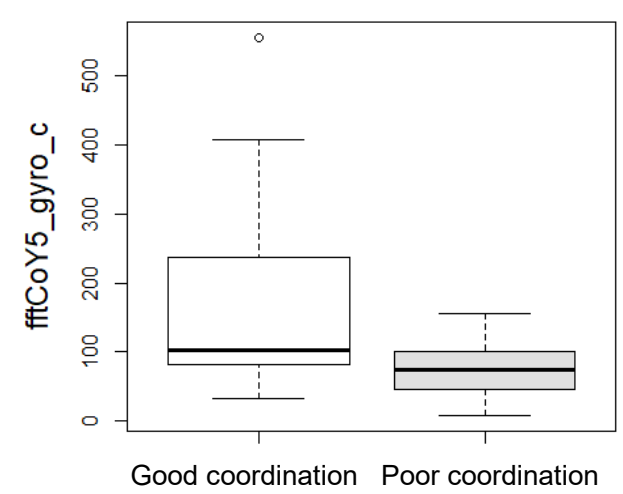

(e)

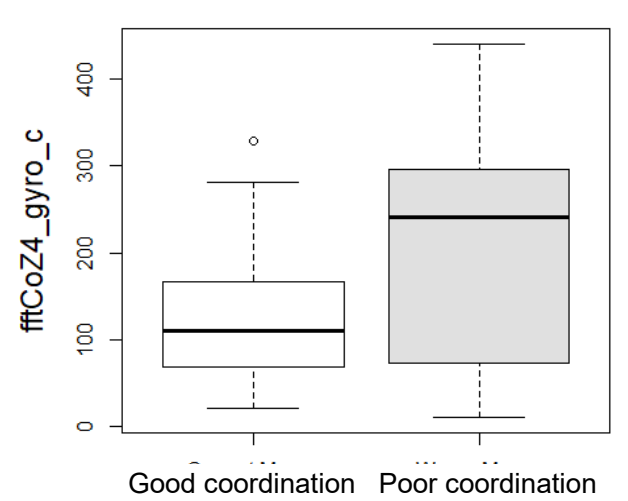

(b)

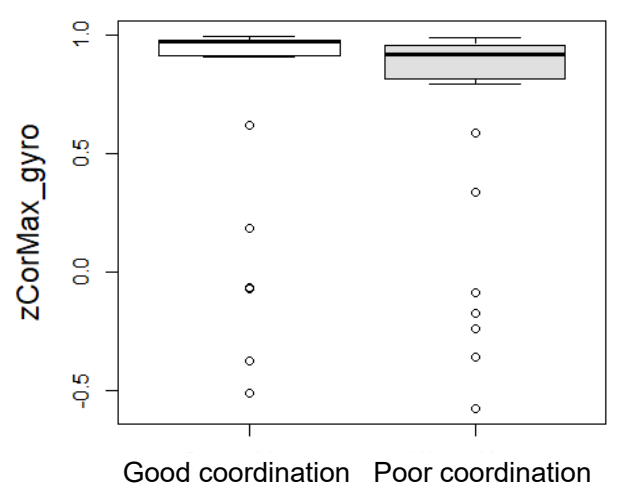

(d)

Figure 6. Box-and-Whisker plots on top five features selected in Method-9 for good and poor coordination during chest rotation: (a) maximal value of cross-correlation of y component of angular velocity between chest and pelvis; (b) fourth FFT coefficient of $z$ component of angular velocity of chest; (c) mean value of $x$ component of angular velocity of pelvis; (d) maximal value of cross-correlation of $z$ component of angular velocity between chest and pelvis; (e) fifth FFT coefficient of y component of angular velocity of chest. Thick lines, bot edges and whiskers represent the median, the $25-75 \%$ quartile range and the overall range respectively. 


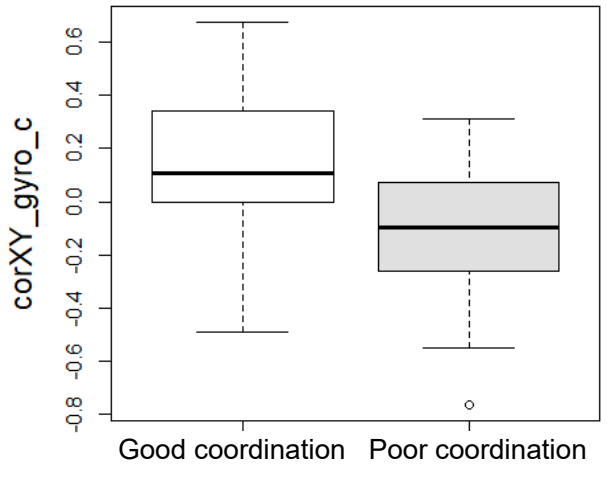

(a)

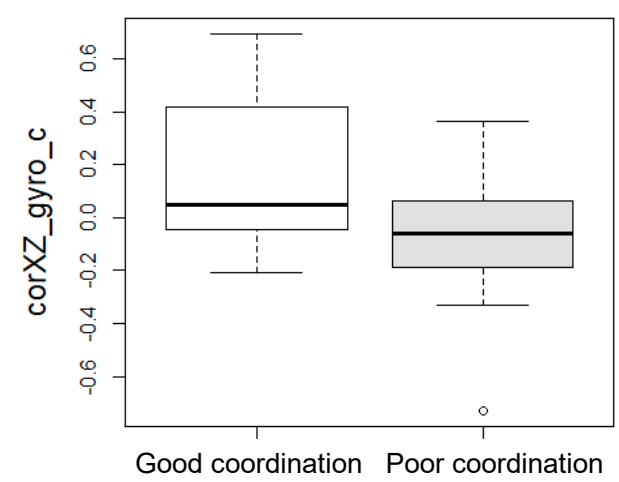

(c)

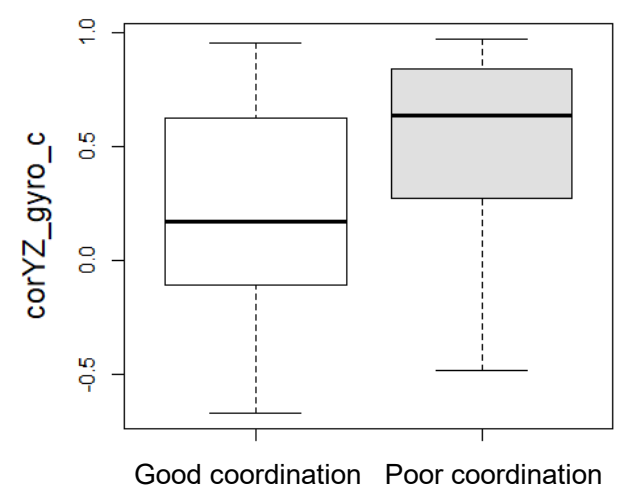

(e)

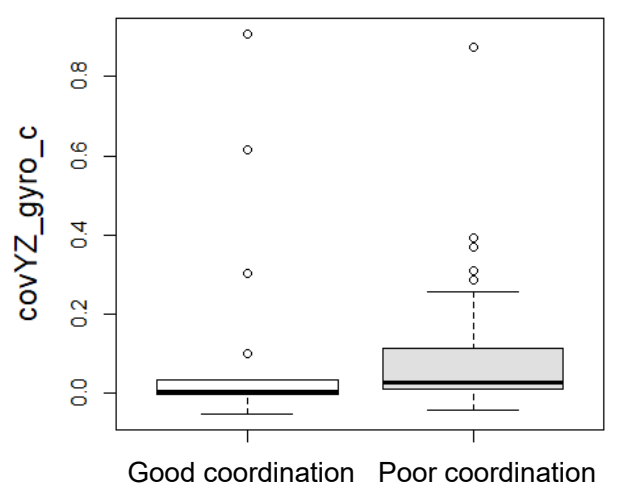

(b)

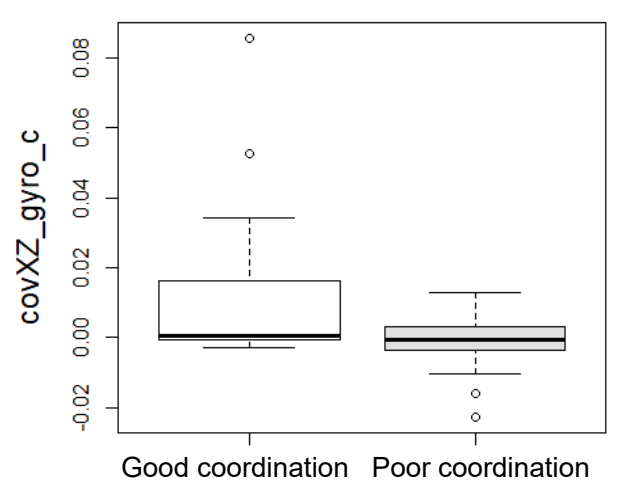

(d)

Figure 7. Box-and-Whisker plots on top five features selected in Method-8 for good and poor coordination during pelvis rotation: (a) correlation between $\mathrm{x}$ and $\mathrm{y}$ components of angular velocity of chest, (b) covariance between $\mathrm{y}$ and $z$ components of angular velocity of chest, (c) correlation between $x$ and $z$ components of angular velocity of chest, (d) covariance between $x$ and $z$ components of angular velocity of chest, and (e) correlation between $y$ and $z$ components of angular velocity of chest.

The Wilcoxon Signed Rank test found statistically significant differences between participants with good coordination and those with poor coordination on the mean values of the top five features. The Box-andWhisker plots further identified the differences in terms of the distribution of the feature values between good and poor coordination. Poor coordination during chest rotation was associated to lower values of the maximal cross- correlation ( $y$ component), to lower values of the mean value ( $x$ component at pelvis), and to lower values of the fifth FFT coefficient ( $y$ component at chest) of angular velocity, while poor coordination during pelvis rotation was associated to weaker correlation between the $x$ and the $y$ components, and to weaker correlation between the $x$ and the $z$ components of angular velocity of chest. These findings both echoed and extended previous findings of 
reduced relative motion between pelvis and trunk during walking and running for people with poor coordination [5, $6,11]$. Additionally, features with strong distinguishing power were identified in three orthogonal directions, suggesting that coordination assessment should consider all three planes of motion as has been advocated in previous studies [11, 15, 31-33]. Another interesting finding was that standard deviations that were widely used to characterise movements variability were not always important features, despite of its wide use in gait studies [51]. This backs up previous findings that variability may not be a proper metrics for assessing the stability of human movement [39].

Although this study obtained promising results, there were some limitations demanding further efforts. The main limitation of this study was that we only constructed global models for the whole cohort. These models thus did not address the characteristics of different populations in terms of age, gender, and exercise habit. Second, the analysis was conducted based on the assumption that interpersonal variation and intrapersonal variation satisfied the same distribution, as the collected datasets contained multiple samples from each participant. This assumption can be eliminated when more participants were recruited in future studies.

\section{Conclusions}

In this study we proposed a pervasive sensing approach for automatic assessment of trunk coordination during chest rotation and pelvis rotation. We have shown that using multiple mobile devices with both sensing modalities (i.e. accelerometer and gyroscope) achieved the best accuracy in classifying trunk coordination during chest rotation $(96 \%)$ and pelvis rotation (100\%). SVM and KNN produced comparable performance while decision tree was the weakest classification technique. Through post hoc analysis, we found that poor coordination during chest rotation was associated to weaker coupling between chest and pelvis and lower angular velocity of the chest, and poor coordination during pelvis rotation was associated to weaker coupling between the three components of angular velocity of the chest. We also found that using single device produced comparable performance for chest rotation (95\%) and slightly reduced performance for pelvis rotation (79\%). Nevertheless, single-device strategy may be a good tradeoff between feasibility and accuracy in practical situation. In summary, our results validated the potential of harnessing widely available mobile devices for automatic nominal assessment of trunk coordination in daily life settings.

\section{Acknowledgements.}

This study was supported by an Artificial Intelligence Research Grant from the New Energy and Industrial Technology Development Organization of Japan. We would like to thank Mr Naoki Koyama and Ms Mio Suzuki for helping with the data collection. We also appreciate the contribution of all participants to this study.

\section{References}

[1] Zimmerman, S, Greene, A, Sloane, P., et al. (2017) Preventing falls in assisted living: results of a quality improvement pilot study. Geriatric Nursing 38(3):185-191.

[2] Suzuki, N. and Endo, S. (1983) A quantitative study of trunk muscle strength and fatigability in the low-back-pain syndrome. Spine 8(1):69-74.

[3] Borghuis, A.J., Hof, A.L. and Lemmink, K. A. (2008) The importance of sensory-motor control in providing core stability. Sports Medicine 38(11): 893-916.

[4] Cresswell, A., Oddsson, L. I. E. and Thorstensson, A. (1994) The influence of sudden perturbations on trunk muscle activity and intra abdominal pressure while standing. Experimental Brain Research 98(2):336-341.

[5] Seay, J., Sauer, S., Patel, T. and Roy, T. (2016) A history of low back pain affects pelvis and trunk coordination during a sustained manual materials handling task. Journal of Sport and Health Science 5(1): 52-60.

[6] Seay, J., Van Emmerik, R. and Hamill, J. (2011) Influence of low back pain status on pelvis-trunk coordination during walking and running. Spine 36(16): 1070-1079.

[7] Selles, R., Wagenaar, R., Smit, T. and Wuisman, P. (2001) Disorders in trunk rotation during talking in patients with low back pain: a dynamical systems approach. Clinical Biomechanics 16(3):175-181.

[8] Shaikh, T., Goussev, V., Feldman, A. and Levin, M. (2013) Arm-trunk coordination for beyond-the-reach movements in adults with stroke. Neurorehabilitation and Neural Repair 28(4):355-366.

[9] Paul, S., Dibble, L., Walther, R., et al. (2017) Characterization of head-trunk coordination deficits after unilateral vestibular hypofunction using wearable sensors. JAMA Otolaryngol Head Neck Surg 143(10):1008-1014.

[10] Esola, M., McClure, P., Fitzgerald, K. and Siegler, S. (1996) Analysis of lumbar spine and hip motion during forward bending in subjects with and without a history of low back pain. Spine 21(1): 71-78.

[11] Seay, J., Van Emmerik, R. and Hamill, J. (2011) Low back pain status affects pelvis-trunk coordination and variability during walking and running. Clinical Biomechanics 26: 572-578.

[12] McIntyre, D., Glover, L., Conino, M., et al. (1991) A comparison of the characteristics of preferred low-back motion of normal subjects and low-back-pain patients. Journal of Spinal Disorders 4(1): 90-95.

[13] Sung, P. (2014) A Kinematic analysis for shoulder and pelvis coordination during axial trunk rotation in subjects with and without recurrent low back pain. Gait \& Posture 40:493-498.

[14] Seay, J., Van Emmerik, R. and Hamill, J. (2014) Trunk bend and twist coordination is affected by low back pain status during running. European Journal of Sport Science 14(6):563-568.

[15] Smith, J. A. and Kulig, K. (2016) Trunk-pelvis coordination during turning: a cross sectional study of young adults with and without a history of low back pain. Clinical Biomechanics 36:58-64.

[16] LeMoyne, R. and Mastroianni, T. (2018) The rise of inertial measurement units. Wearable and Wireless 
Systems for Healthcare I. In Smart Sensors, Measurement and Instrumentation (Singapore: Springer).

[17] Iosa, M., Picerno, P., Paolucci, S. and Morone, G. (2016) Wearable inertial sensors for human movement analysis. Expert Review of Medical Devices 13(7): 641-659.

[18] Mukhopadhyay, S. C. (2015) Wearable sensors for human activity monitoring: a review. IEEE Sensors Journal 15(3):1321-1330.

[19] Shany, T., Redmond, S., Narayanan, M. and Lovell, N. (2012) Sensors-based wearable systems for monitoring of human movement and falls. IEEE Sensors Journal 12(3):658-670.

[20] Allet, L., Knols, R. H., Shirato, K. and De Bruin, E. D. (2010) Wearable Systems for Monitoring Mobility-Related Activities in Chronic Disease: A Systematic Review. Sensors 10(10): 9026-9052.

[21] Liang, Z., Nishimura, T., Iino, N., Yoshida, Y. and Nishimura, S. (2017) Utilizing mobile devices for evaluating body trunk coordination: Feasibility and preliminary results. In Proceedings of the 10th International Conference on Mobile Computing and Ubiquitous Network (ICMU), Toyama, Japan.

[22] Liang, Z., Nishimura, T., Iino, N., Yoshida, Y. and Nishimura, S. (2017) Novel use of Nintendo Wii Balance Board for automatic evaluation on motion quality. In Proceedings of the 6th IEEE Global Conference on Consumer Electronics (GCCE), Nagoya, Japan.

[23] Liang, Z. and Chapa-Martell, M. A. (2019) Achieving accurate ubiquitous sleep sensing with consumer wearable activity wristbands using multi-class imbalanced classification. In Proceedings of the 17th IEEE Intl Conf Perv Intel Comp (PICOM 2019), Fukuoka, Japan.

[24] Liang, Z., and Chapa-Martell, M. A. (2019) Combining resampling and machine learning to improve sleepwake detection of Fitbit wristbands. In Proceedings of the 7th International Conference on Healthcare Informatics, Xi'an, China.

[25] Kaur, P. and Sharma, M. (2019) Diagnosis of human psychological disorders using supervised learning and nature-inspired computing techniques: a meta-analysis. Journal of Medical Systems 43:204.

[26] Sharma, M., Singh, G. and Singh, R. (2017) Stark assessment of lifestyle based human disorders using data mining based learning techniques. IRBM 38(6):305-324, 2017.

[27] Altmann, V., Groen, B., Groenen, K., et al. (2016) Construct validity of the trunk impairment classification system in relation to objective measures of trunk impairment. Archives of Physical Medicine and Rehabilitation 97(3): 437-444.

[28] Kim, J., Parnianpour, M. and Marras, W. (1996) Quantitative assessment of the control capability of the trunk muscles during oscillatory bending motion under a new experimental protocol. Clinical Biomechanics 11(7): 385-391.

[29] Caliandro, P., Iacovelli, C., Conte, C., et al. (2017) Trunklower limb coordination pattern during gait in patients with ataxia. Gait \& Posture 57:252-257.

[30] Marras, W., Ferguson, S., Purnendu, G. and Bose S. (1999) The quantification of low back disorder using motion measures: methodology and validation. Spine 24(20): 2091

[31] Sung, P. and Kim, Y. H. (2011) Kinematic analysis of symmetric axial trunk rotation on dominant hip. Journal of Rehabilitation Research \& Development 48(8): 1029-1036.
[32] Lee, N.-G., Lee, J.-A. and Kim, J.-B. (2013) A comparison of three-dimensional spine kinematics during multidirectional trunk movement between elderly subjects with degenerative spine disease and healthy young adults. J Phys Ther Sci 25:21-26.

[33] Van Emmerik, R., McDermott, W., Haddad, J. and Van Wegen E. (2005) Age-related changes in upper body adaptation to walking speed in human locomotion. Gait \& Posture 22(3):233-239.

[34] Lund, T., Nydegger, T., Schlenzka, D. and Oxland, T. (2002) Three-dimensional motion patterns during active bending in patients with chronic low back pain. Spine 27(17):1865-1874.

[35] Macedo, P., Afonso, J. and Simoes, R. (2015) A wireless posture monitoring system for personalized home-based rehabilitation. Sensors \& Transducers 6:41-51.

[36] Hamill, J., Haddad, J. and McDermott, W. (2000) Issues in quantifying variability from a dynamical systems perspective. Journal of Applied Biomechanics 16:407-418.

[37] Haddad, J., Van Emmerik, R., Whittlesey, S. and Hamill, J. (2006). Adaptations in interlimb and intralimb coordination to asymmetrical loading in human walking. Gait \& Posture 23(4):429-434.

[38] Burgess-Limerick, R., Abernethy, B. and Neal, R. (1993) Relative phase quantifies interjoint coordination. Journal of Biomechanics 26(1):91-94.

[39] Van Emmerik, R. and Van Wegen, E. (2000) On variability and stability in human movement. Journal of Applied Biomechanics 16:394-406.

[40] Jarvis, D., Smith, J. A. and Kulig, K. (2014) Trunk coordination in dancers and nondancers. Journal of Applied Biomechanics 30:547-554.

[41] Cappozzo, A., Croce, U. D., Leardini, A. and Chiari, L. (2005) Human movement analysis using stereophotogrammetry: Part 1: theoretical background. Gait \& Posture 21(2):186-196.

[42] Kim, M. C. and Eng, J. J. (2004) Magnitude and pattern of 3D kinematic and kinetic gait profiles in persons with stroke: relationship to walking speed. Gait \& Posture 20(2): 140-146.

[43] Kavanagh, J. J. and Menz, H. B. (2008) Accelerometry: a technique for quantifying movement patterns during walking. Gait \& Posture 28(1): 1-15.

[44] Mayagoitia, R. E., Nene, A. V. and Veltink, P. H. (2001) Accelerometer and rate gyroscope measurement of kinematics: an inexpensive alternative to optical motion analysis systems. Journal of Biomechanics 35(4):537-542.

[45] Iosa, M., Morone, G., Fusco, A., et al. (2012) Effects of walking endurance reduction on gait stability in patients with stroke. Stroke Research and Treatment 2012:810415.

[46] Iosa, M., Morelli, D., Marro, T., et al. (2013) Ability and stability of running and walking in children with cerebral palsy. Neuropediatrics 44(3):147-154.

[47] Menz, H. B., Lord, S. R. and Fitzpatrick, R. C. (2003) Acceleration patterns of the head and pelvis when walking on level and irregular surfaces. Gait \& Posture 18(1):35-46.

[48] Dingwell, J. B. and Marin, L. C. (2006) Kinematic variability and local dynamic stability of upper body motions when walking at different speeds. Journal of Biomechanics 39(3): 444-452.

[49] Terrier, P. and Deriaz, O. (2011) Kinematic variability, fractal dynamics and local dynamic stability of treadmill walking. Journal of NeuroEngineering and Rehabilitation 8: 12 .

[50] Aziz, O. and Robinovitch, S. N. (2011) An analysis of the accuracy of wearable sensors for classifying the causes of 
falls in humans. IEEE Transactions on Neural Systems and Rehabilitation Engineering 19(6):670-676.

[51] Hamacher, D., Singh, N., Van Dieen, J., et al. (2011) Kinematic measures for assessing gait stability in elderly individuals: a systematic review. Journal of the Royal Society Interface 8:1682-1698.

[52] Marigold, D. and Patla, A. (2008) Age-related changes in gait for multi-surface terrain. Gait \& Posture 27(4):689696.

[53] Iosa, M., Mazza, C., Pecoraro, F., et al. (2010) Control of the upper body movements during level walking in patients with facioscapulohumeral dystrophy. Gait \& Posture 31(1):68-72.

[54] Cappozzo, A. (1982) Low frequency self-generated vibration during ambulation in normal men. Journal of Biomechanics 15:8.

[55] Mazza, C., Iosa, M., Pecoraro, F. and Cappozzo, A. (2008) Control of the upper body accelerations in young and elderly women during level walking. Journal of NeuroEngineering and Rehabilitation 5:30.

[56] Kan, Y.-C. and Chen, C.-K. (2012) A wearable inertial sensor node for body motion analysis. IEEE Sensors Journal 12(3):651-657.

[57] Cheng, J., Amft, O., Bahle, G. and Lukowicz, P. (2013) Designing sensitive wearable capacitive sensors for activity recognition. IEEE Sensors Journal 13(10):39353947.

[58] Stikic, M., Larlus, D., Ebert, S. and Schiele, B. (2011) Weakly supervised recognition of daily life activities with wearable sensors. IEEE Transactions on Pattern Analysis and Machine Intelligence 33(12): 2521-2537.

[59] Ayzenberg, Y. and Picard, R. W. (2014) FEEL: a system for frequent event and electrodermal activity labelling. IEEE Journal of Biomedical and Health Informatics 18(1): 266-277.

[60] Wu, W., Bui, A. A., Batalin, M. A., et al. (2007) Incremental diagnosis method for intelligent wearable sensor systems. IEEE Transactions on Information Technology in Biomedicine 11(5): 553-562.

[61] Hegde, N., Bries, M., Swibas, T., et al. (2018) Automatic recognition of activities of daily living utilizing insolebased and wrist-worn wearable sensors. IEEE Journal of Biomedical and Health Informatics 22(4): 979-988.

[62] Liang, Z. and Chapa-Martell, M. A. (2019) Accuracy of Fitbit wristbands in measuring sleep stage transitions and the effect of user-specific factors. JMIR Mhealth Uhealth 7(6): e13384.

[63] Liang, Z. and Chapa-Martell, M. A. (2019) Combining numerical and visual approaches in validating sleep data quality of consumer wearable wristbands. In Proceedings of International Workshop on Information Quality and Quality of Service for Pervasive Computing, Kyoto, Japan.

[64] Liang, Z. Ploderer, B., Chapa-Martell, M. A. (2017) Is fitbit fit for sleep-tracking?: sources of measurement errors and proposed countermeasures. In Proceedings of the 11th EAI International Conference on Pervasive Computing Technologies for Healthcare, 476-479, Barcelona, Spain.

[65] Liang, Z. and Chapa-Martell, M. A. (2018) Validity of consumer activity wristbands and wearable EEG for measuring overall sleep parameters and sleep structure in free-living conditions. Journal of Healthcare Informatics Research 2 (1-2):152-178.

[66] Cooper, A., Page, A., Fox, K. and Mission, J. (2000) Physical activity patterns in normal, overweight and obese individuals using minute-by-minute accelerometry. Eur $J$ Clin Nutr 54(12): 887-894.
[67] De Bruin, E. D., Hartmann, A., Uebelhart, D., et al. (2008) Wearable systems for monitoring mobility-related activities in older people: a systematic review. Clin Rehabil 22:878-895.

[68] Hobert, M., Maetzler, W., Aminian, K. and Chiari, L. (2014) Technical and clinical view on ambulatory assessment in Parkinson's disease. Acta Neurologica Scandinavica 130(3): 139-147.

[69] Haeuber, E., Shaughnessy, M., Forrester, L., et al. (2004) Accelerometer monitoring of home- and community-based ambulatory activity after stroke. Archives of Physical Medicine and Rehabilitation 85(12): 1997-2001.

[70] Hinton-Lewis, C., McDonough, E., Moyle, G. and Thiel, D. (2016) An assessment of postural sway in ballet dancers during first position, releve and saute with accelerometers. Procedia Engineering 147:127-132.

[71] Mathie, M., Coster, A., Lovell, N. and Celler, B. (2004) Accelerometry: providing an integrated, practical method for long-term, ambulatory monitoring of human movement. Physiological Measurement 25(2): R1-20.

[72] Veltink, P. H., Bussmann, H. B., De Vries, W., et al. (1996) Detection of static and dynamic activities using uniaxial accelerometers. IEEE Transactions on Rehabilitation Engineering 4(4): 375-385.

[73] Trost, S. G., Fees, B. S., Haar, S. J., et al. (2012) Identification and Validity of Accelerometer Cut - Points for Toddlers. Obesity 20(11):2317-2319.

[74] Freedson, P., Pober, D. and Janz, K. F. (2005) Calibration of accelerometer output for children. Med Sci Sports Exerc 37: S523-530.

[75] Fischer, C., Yildirim, M., Salmon, J. and Chinapaw, M. J. (2012) Comparing different accelerometer cut-points for sedentary time in children. Pediatric Exercise Science 24:220-228.

[76] Kim, Y., Beets, M. W. and Welk, G. J. (2012) Everything you wanted to know about selecting the "right" Actigraph accelerometer cut-points for youth, but...: A systematic review. Journal of Science and Medicine in Sport 15(4): 311-321.

[77] Hickey, A., Newham, J., Slawinska, M., et al. (2016) Estimating cut points: a simple method for new wearables. Maturitas 83:78-82.

[78] Matthew, C. E. (2005) Calibration of accelerometer output for adults. Med Sci Sports Exerc 37: S512-522.

[79] Dijkstra, B., Kamsma, Y. and Zijlstra, W. (2010) Detection of gait and postures using a miniaturized triaxial accelerometer-based system: accuracy in patients with mild to moderate Parkinson's disease. Archives of Physical Medicine and Rehabilitation 91(8): 1272-1277.

[80] Molt, R. W., Snook, E. M., Agiovlasitis, S. and Suh, Y. (2009) Calibration of accelerometer output for ambulatory adults with multiple sclerosis. Archives of Physical Medicine and Rehabilitation 90(10): 1778-1784.

[81] Nero, H., Wallen, M. B., Franzen, E., et al. (2015) Accelerometer cut points for physical activity assessment of older adults with Parkinson's disease. PLoS One 10(9): e0135899.

[82] Bourke, A., O'Donovan, K. and OLaighin, G. (2008) The identification of vertical velocity profiles using an inertial sensor to investigate pre-impact detection of falls. Medical Engineering \& Physics 30:937-946, 2008.

[83] Klenk, J., Becker, C., Lieken, F., et al. (2011) Comparison of acceleration signals of simulated and real-world backward falls. Med Eng Phys 33:368-373. 
[84] Dingwell, J. B. and Cusumano, J. (2000) Nonlinear time series analysis of normal and pathological human walking. Chaos 10(4):848-863.

[85] Nishimura, T., Liang, Z., Nishimura, S., et al. (2017) Axis Visualizer: enjoy core torsion and be healthy for health promotion community support. New Frontiers in Artificial Intelligence, 380-392.

[86] Schettlinger, K., Fried, R. and Gather, U. (2010) Real-time signal processing by adaptive repeated median filters. Int $J$ Adapt Control Signal Process 24(5): 346-362.

[87] Machado, I., Gomes, L., Gamboa, H., et al. (2015) Human activity data discovery from triaxial accelerometer sensor: non-supervised learning sensitivity to feature extraction parametrization. Information Processing \& Management 51(2): 204-214.

[88] Karantonis, D., Narayanan, M., Mathie, M., et al. (2006) Implementation of a real-time human movement classifier using a triaxial accelerometer for ambulatory monitoring. IEEE Transaction on Information Technology in Biomedicine 10(1):156-167.

[89] Phinyomark, A., Phukpattaranont, P. and Limsakul, C. (2012) Feature reduction and selection for EMG signal classification. Expert Systems with Applications 39(8): 7420-7431.

[90] Preece, S., Goulermas, J. Y., Kenney, L. and Howard, D. (2009) A comparison of feature extraction method for the classification of dynamic activities from accelerometer data. IEEE Transactions on Biomedical Engineering 56:871-879.

[91] Rafal, S., Janusz, M., Wieslaw, O. and Robert, S. (2011). Test-retest reliability of measurements of the centre of pressure displacement in quiet standing and during maximal voluntary body leaning among healthy elderly men. J Hum Kinet 28:15-23.

[92] Li, Q., Chen, H., Huang, H., Zhao, X., et al. (2017) An enhanced Grey Wolf optimization based f eature selection wrapped kernel extreme learning machine for medical diagnosis. Computational and Mathematical Methods in Medicine 2017, Article ID 9512741.

[93] Li, Y., Alexander, M., Glazebrook, C. and Leiter, J. (2016) Quantifying inter-segmental coordination during the instep soccer kicks. Int J Exerc Sci 9(5): 646-656.

[94] Kim, Y. K., Kim, Y. H. and Im, S. J. (2011) Inter-joint coordination in producing kicking velocity of taekwondo kicks. J Sports Sci Med 10(1): 31-38.
[95] Chan, J., Leung, H. and Poizner, H. (2010) Correlation among joint motions allows classification of parkinsonian versus normal 3D reaching. IEEE Trans Neural Syst Rehabil Eng 18(2): 142-149.

[96] Seifert, L., Leblanc, H., Chollet, D. and Delignieres, D. (2010) Inter-limb coordination in swimming: effect of speed and skill level. Human Movement Science 29(1): 103-113.

[97] Tuncel, O., Altun, K. and Barshan, B. (2009) Classifying human leg motions with uniaxial piezoelectric gyroscopes. Sensors 9(11): 8508-8546.

[98] Antoniadis, A., Lambert-Lacroix, S. and Leblanc, F. (2003) Effective dimension reduction methods for tumor classification using gene expression data. Bioinformatics 19(5): 563-570.

[99] Friedman, J. (1997) On bias, variance, 0/1-Loss, and the curse-of-dimensionality. Data Mining and Knowledge Discovery 1(1): 55-77.

[100] Granitto, P., Furlanello, C., Biasioli, F. and Gasperi, F. (2006) Recursive feature elimination with random forest for PTR-MS analysis of agroindustrial products. Chemometrics and Intelligent Laboratory Systems 83(2): 83-90.

[101]Osei-Bryson, K.-M. and Giles, K. (2006) Splitting methods for decision tree induction: an exploration of the relative performance of two entropy-based families. Information Systems Frontiers 8(3): 195-209.

[102]Altman, D. and Bland, M. (1994) Diagnostic tests 1: sensitivity and specificity. $B M J$ 308(6943): 1552 .

[103]Altman, D. and Bland, M. (1994) Diagnostic tests 2: predictive values. British Medical Journal 309:102.

[104]Velez, D., White, B., Motsinger, A., et a. (2007) A balanced accuracy function for epistasis modelling in imbalanced datasets using multifactor dimensionality reduction. Genetic Epidemiology 31(4):306-315s.

[105]Wilcoxon, F. (1945) Individual comparisons by ranking methods. Biometrics Bulletin 1(6):80-83.

[106]Benjamini, Y. (1988) Opening the Box of a Boxplot. The American Statistician 42(4): 257-262.

[107]Kuhn, M. (2008) Building predictive models in R using the caret package. Journal of Statistical Software 28(5): 1-26.

[108] Shapiro, S. and Wilk, M. (1965) An analysis of variance test for normality (complete samples). Biometrika 52(34):591-611. 